\title{
Options for thumb revascularization: our experience and literature review
}

\author{
Pradeoth Mukundan Korambayil', Prashanth Varkey Ambookan', Vinoth Kumar Dilliraj² \\ ${ }^{1}$ Department of Plastic Surgery and Burns, Jubilee Institute of Surgery for Hand, Aesthetic and Microsurgery, Jubilee Mission Medical \\ College and Research Institute, P.B. No.737, Thrissur 680005, Kerala, India. \\ ${ }^{2}$ Department of Plastic Surgery, Vijaya Hospital, Chennai, India.
}

Address for correspondence: Dr. Pradeoth Mukundan Korambayil, Assistant Professor, Jubilee Institute of Surgery for Hand, Aesthetic and Microsurgery, Jubilee Mission Medical College and Research Institute, Thrissur 680005, Kerala, India. E-mail: pradeoth@gmail.com

\begin{abstract}
Traumatic injuries to the thumb resulting in segmental loss of a digital artery are not uncommon. To bridge the gap and repair a transacted digital artery, the superficial palmar arch or the radial digital artery of the index finger can be used to reconstruct the ulnar digital artery of thumb for revascularization. Revascularization following segmental loss resulting from a crushed ulnar digital artery of the thumb can be performed based on the superficial palmar arch or the radial digital artery of the index finger, avoiding anastomosis at two sites and hence providing better results. The digital vein from the index finger can also be used to enhance the venous return of the injured thumb. However, because of known variability in the palmar arch, intraoperative verification is needed to ensure the safe transfer of the arch or the radial digital artery of the index finger. The aim of this article is to discuss the possibilities for thumb revascularization, using a case report in which the injured thumb was revascularized with a superficial palmar arch.
\end{abstract}

Key words:

Radial digital artery index finger, superficial palmar arch, thumb revascularization, ulnar digital artery thumb

\section{INTRODUCTION}

The decision whether to proceed with revascularizing the thumb following a crush injury depends on several factors, including the mechanism of injury, the patient's age, occupation and hand dominance, and overall medical condition and intraoperative assessment of the injured structures. Crush injuries at the base of the thumb involving loss of digital artery segments can present a

\begin{tabular}{|l|l|}
\hline \multicolumn{2}{|c|}{ Access this article online } \\
\hline Quick Response Code: & Website: \\
\hline & www.parjournal.net \\
\cline { 2 - 2 } & \\
\hline & \\
\hline
\end{tabular}

challenge to the reconstructive surgeon. Primary repair of the digital artery may be difficult and may be achieved by shortening the proximal phalangeal bone if there are associated fractures. Repair of the digital artery is likely to require vein grafting, as significant segmental loss of the digital artery often results from injuries in this area. To bridge the gap and repair a transected digital artery, the palmar arch or radial digital artery of the index finger can be used to reach the ulnar digital artery of the thumb without a vein graft. In addition, a vein from the index finger can be used to achieve venous drainage of the thumb.

\section{CASE REPORT}

A 60-year-old male sustained a mutilating injury to his dominant right thumb [Figures 1-3]. A radiograph of the right hand in the anteroposterior view showed a fracture 
at the level of the shaft of the proximal phalanx of the thumb [Figure 4]. Intraoperatively, transection of the ulnar digital artery was at the level of the base of the thumb. The thumb was ischemic. Skeletal fixation was achieved using Kirschner wires [Figure 5]. Dissection of the digital nerve revealed contused nerve fibers, although the fibers were intact. The ulnar digital artery was found to be transected at the shaft level with segmental loss; this segmental loss made primary repair difficult. The radial digital artery and the nerve of the thumb were contused but intact. The dorsal veins were contused.

To bridge the gap, the superficial palmar arch was dissected until its ulnar end where the branch to the common digital artery to the index and middle fingers arising from the palmar arch. A digital clamp was placed over the ulnar end of the palmar arch and the radial digital artery of the index finger. The tourniquet was released and the vascularity of the index finger was confirmed, before reapplying the tourniquet. A Vascular clip was used to ligate the superficial palmar arch just before the division of common digital artery to the index and middle fingers. To obtain additional length, the radial digital artery to the index finger was also ligated and divided. The palmar arch was turned to reach the ulnar digital

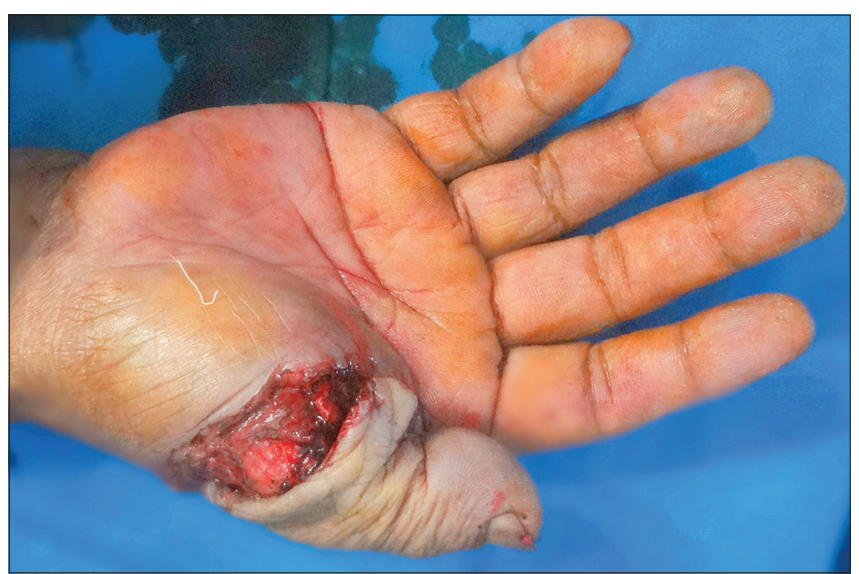

Figure 1: Crush injury to the base of the right thumb, volar aspect

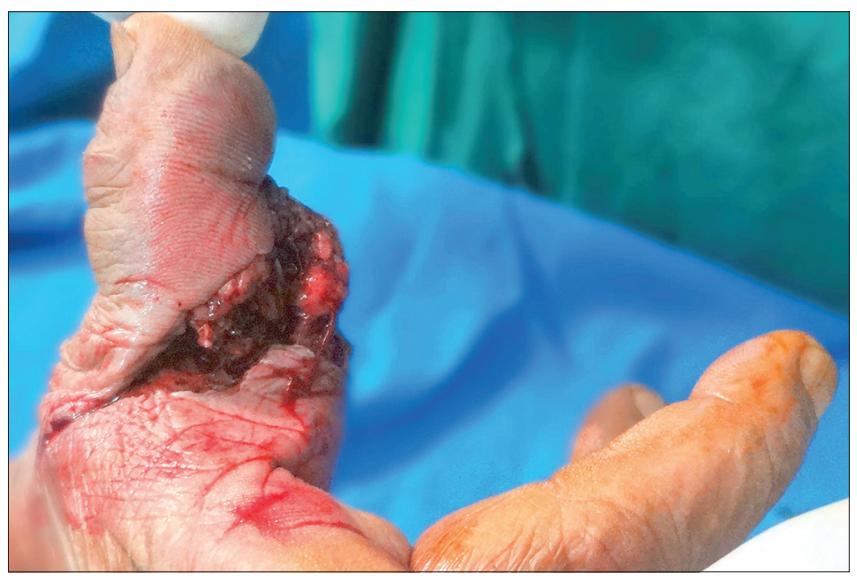

Figure 3: Ulnar aspect of the injured thumb artery of the thumb, facilitating end-to-end repair without a vein graft. The digital vein of the index finger was also utilized to allow venous drainage of the thumb [Figure 6]. On release of the tourniquet, there was good blood flow into the thumb and fingers [Figure 7]. Postoperatively, the patient received six sessions of hyperbaric oxygen therapy. After an uneventful hospital course, the patient was discharged on the tenth postoperative day.

\section{DISCUSSION}

Avulsion injuries to the thumb can result in extensive damage to long segments of vessels, which makes direct suturing of the structures difficult. The decision to proceed with revascularizing an avulsed thumb depends on several factors, including the mechanism of injury, the patient's age, occupation and hand dominance, and overall medical condition and intraoperative assessment of the injured structures. When the decision is taken to retain the thumb by revascularization, the options available are to use a vein graft to reconstruct the segmental loss or to transfer nearby vessels to adequately bridge the gap of the injured

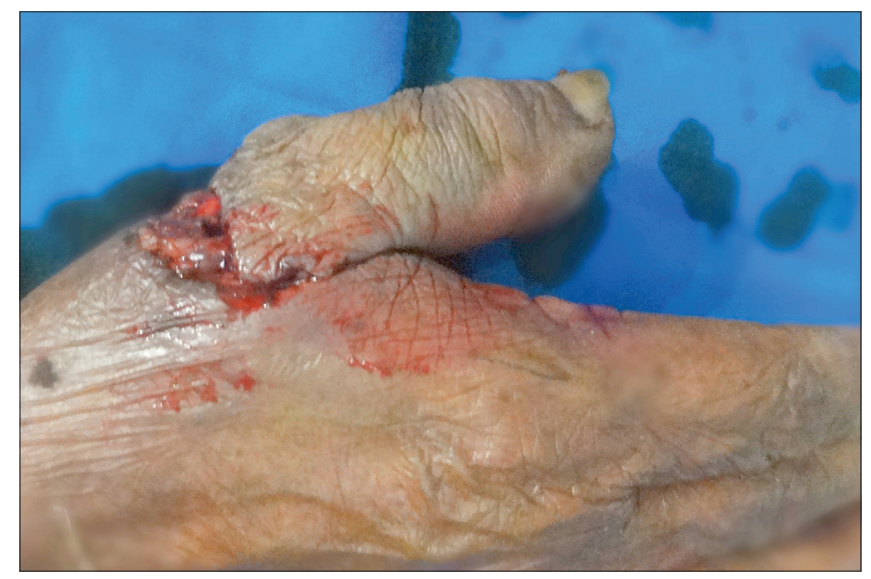

Figure 2: Dorsal aspect of the injured thumb

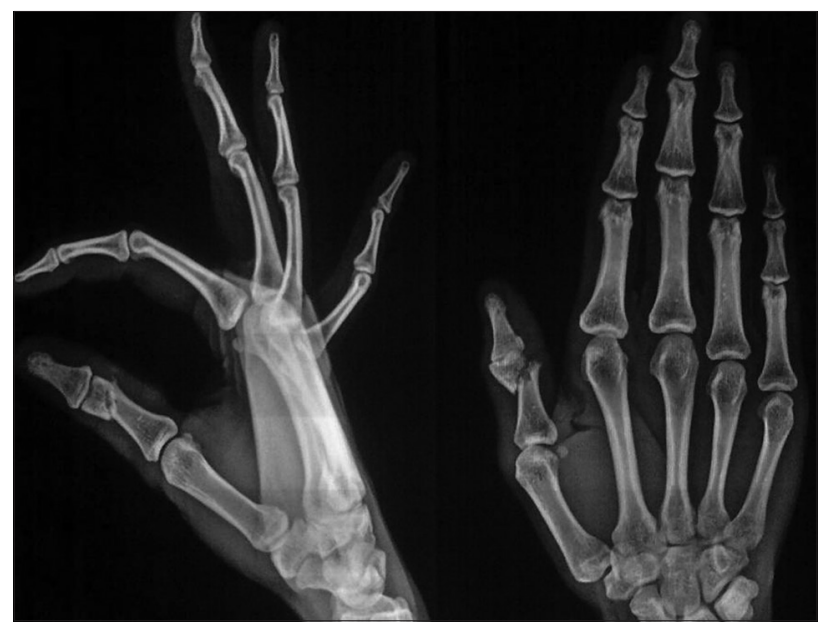

Figure 4: Radiograph of the right hand in the antero-posterior view, showing a fracture at the level of shaft of the proximal phalanx of the thumb 
segment. Ideally, a single end-to-end primary arterial repair of the ulnar digital artery can obviate the need for two anastomotic sites, as in the case of vein grafting.

A classic morphology of the complete superficial palmar arch is formed by the superficial palmar branch of the ulnar artery and the superficial palmar branch of the radial artery. ${ }^{[1]}$ Superficial and deep palmar arches of the hand, formed by the radial and ulnar arteries, provide the dominant blood supply to the hand, with an intricate network of collateral flow. The superficial palmar arch is classified into two categories: complete or incomplete. The latter is formed when the anastomoses between the radial and ulnar superficial vessels constituting the arch are absent..$^{[2]}$

It is important for surgeons dealing with reconstructive hand surgeries and restoration of the functional anatomy of hand to understand how the pattern of the superficial palmar arch can vary. Several cadaveric and radiographic studies have revealed enormous variability in the vascular anatomy of the deep and superficial palmar arches. ${ }^{[3]}$ Conventionally, the superficial palmar branches of the ulnar and radial arteries form the superficial palmar

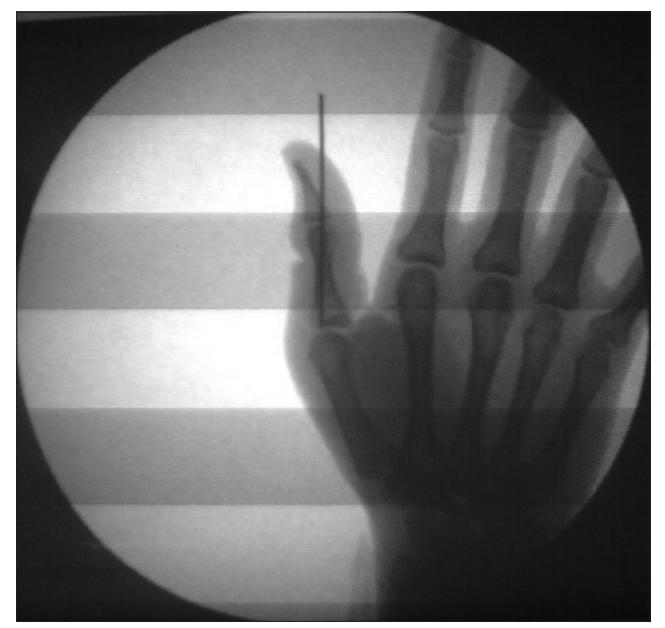

Figure 5: C-arm picture demonstrating skeletal fixation with Kirschner wires

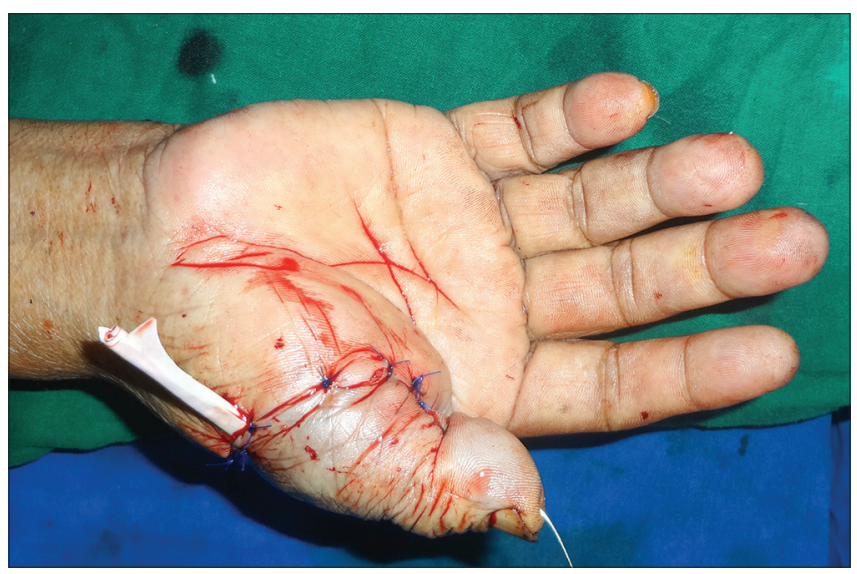

Figure 7: Postoperative picture following revascularization arch. The ulnar artery appears to be the main feeding vessel. ${ }^{[1]}$ The ability to reconstitute the arterial flow when revascularizing the thumb using the palmar arch or radial digital artery of the index finger depends on the anatomic configuration of the superficial palmar arch. The surgeon should always be aware of the variations in the superficial palmar arch before proceeding, and the expendability of the superficial palmar arch or the radial digital artery branch must be established to avoid ischemia of adjacent digits. If the anatomy of the superficial palmar arch is normal [Figure 8], then the palmar arch or the radial digital artery of the index finger can be safely transferred distally to the ulnar digital artery to revascularize the thumb [Figure 9]; however, the possibility of using a reverse radial forearm flap to resurface the palmar or dorsal defect associated with these types of injury may be compromised. Richard and Goldner have discussed transposing the digital neurovascular bundle in patients who have experienced a crush injury of the digits. ${ }^{[4]}$

Other methods used to revascularize such injuries, include reconstructing the injured segment with vein grafts harvested from the distal third of the forearm,

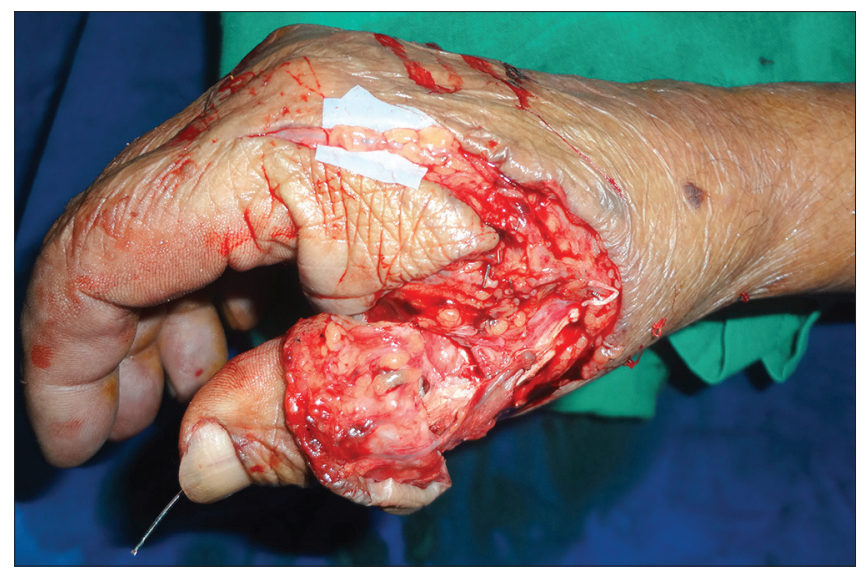

Figure 6: The superficial palmar arch and digital vein from the index finger were used to revascularize the thumb

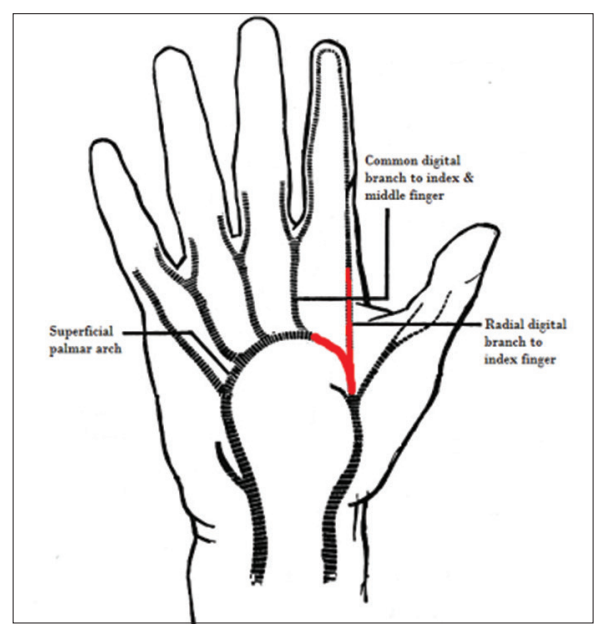

Figure 8: Schematic depicting the normal superficial palmar arch anatomy 


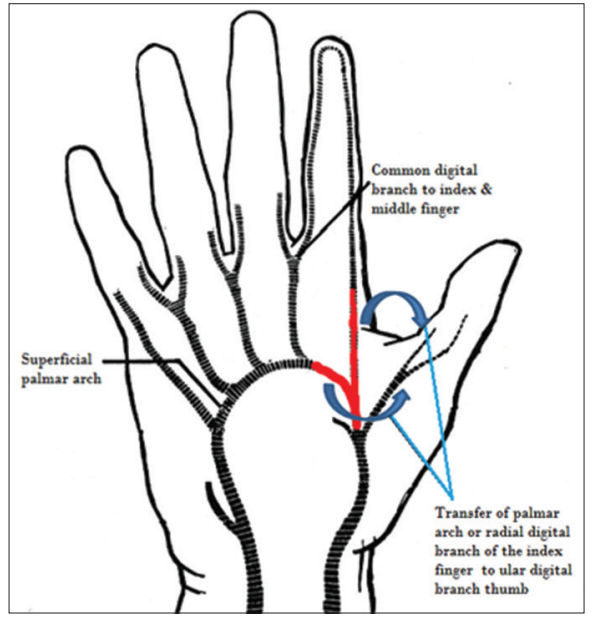

Figure 9: Schematic representing transfer of the palmar arch or radial digital artery of the index finger for revascularization of the thumb

vessel shift of the radial or ulnar arteries, and forming an arteriovenous fistula [Figure 10].

The advantage of the vein graft procedures is that there is no need to sacrifice the palmar arch. The disadvantages are the need for two anastomoses, and that the proximal part of the injured artery can be in spasm since it is near to the zone of trauma. This can result in an unfavorable outcome following anastomosis, with the need for further revision procedures and additional incisions for vein graft harvest. The advantages of the transfer of superficial palmar arch or radial digital artery for thumb revascularization over vein grafts are the need for just one anastomosis, which reduces the incidence of thrombosis, and that the artery borrowed for anastomosis is out of the zone of trauma, resulting in better outcomes. The disadvantage of this procedure is the possibility of loss of the index finger, although the chance of this is low as one vascular bundle is retained and there are additional arterial perforators from the metacarpal arteries.

Another method with which to salvage the thumb following crush or avulsion injuries is to shift the undamaged arteries (radial or ulnar). ${ }^{[4]}$ Disadvantages of this method are scar contracture, size mismatch of the vessels, and concomitant damage to the utilized vessel.

Some authors have suggested creating an arteriovenous fistula. ${ }^{[5]}$ This method is usually used when there is no suitable artery in the devascularized part. Better results are obtained when the volar veins are used for anastomosis and when there is less tension on the anastomosis between the proximal volar digital artery and the dorsal vein. ${ }^{[6]}$ Morris et al. have suggested that the presence or absence of venous valves appears to be an

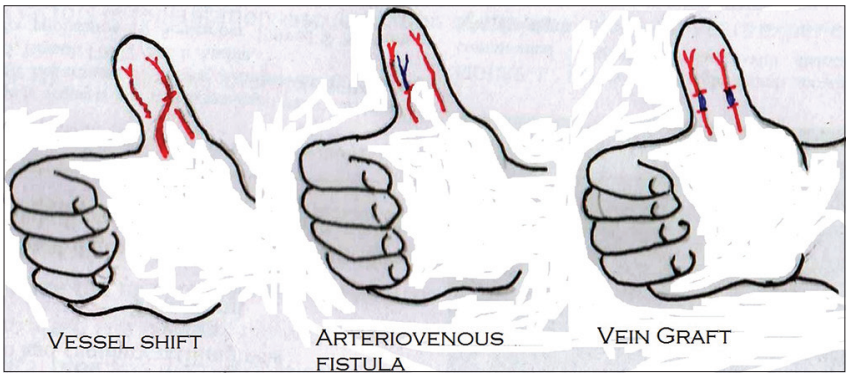

Figure 10: Options for thumb revascularization

important consideration ${ }^{[7]}$ and warrants further evaluation before attempting a clinical transfer.

The principle of transposing the radial digital artery or superficial palmar arch could very well be used in cases of amputation at the level of the base of the thumb, where venous grafts may be avoided for establishing the circulation.

\section{CONCLUSION}

Revascularizing the thumb is a challenging and rewarding experience for the plastic surgeon. Knowledge of the superficial palmar arch and its anatomical variability are critical for successful revascularization. Therefore, bridging the vascular gap to repair the transected ulnar digital artery of the thumb, superficial palmar arch or the radial digital artery of the index finger could be safely transferred distally for revascularisation.

\section{REFERENCES}

I. Hazani R, Elston J, Brooks D,Wilhelmi BJ. Bridging the gap in hand replantation: use of the common digital artery for completion of the superficial palmar arch. Plast Reconstr Surg 2010;126:2037-42.

2. Gellman H, Botte MJ, Shankwiler J, Gelberman RH.Arterial patterns of the deep and superficial palmar arches. Clin Orthop Relat Res 200I;383:4I-6.

3. Dhar P, Lall K. An atypical anatomical variation of palmar vascular pattern. Singapore Med J 2008;49:e245-9.

4. Richard D, Goldner JR. Urbaniak, replantation. In:Wolfe SW, editor. Green's Operative Hand Surgery. 6th ed. Philadelphia, U.S.A: Churchill Livingstone; 2010. p. I59I-2.

5. Hawary MB, Cardoso E, Mahmud S.Afferent arteriovenous fistula in thumb revascularization. Ann Saudi Med 1997; 17:99-100.

6. Inoue G, Tamura Y. The use of an afferent arteriovenous fistula in digit replantation surgery: a report of two cases. Br J Plast Surg 1991;44:230-3.

7. Morris SF, MacGill KA, Taylor GI. Scalp replantation by arterialised venous network flow-through. Br J Plast Surg 1992;45: 187-92.

How to cite this article: Korambayil PM, Ambookan PV, Dilliraj VK. Options for thumb revascularization: our experience and literature review. Plast Aesthet Res 2014;1:37-40.

Source of Support: Nil, Conflict of Interest: None declared.

Received: 27-02-2014; Accepted: 28-05-2014 